The International

Journal of

Integrated

Engineering

\title{
Mechanical Properties of Epoxy Composites Containing Carbon Black and Graphene
}

\author{
R. N. Othman ${ }^{1 *}$, I. A. Rahman', M. K. Faidzi ${ }^{1}$, K. Z. Ku Ahmad ${ }^{1}$ \\ Department of Mechanical Engineering, Faculty of Engineering, \\ Universiti Pertahanan Nasional Malaysia, Kem Sungai Besi, 57000 Kuala Lumpur, MALAYSIA \\ *Corresponding author
}

DOI: https://doi.org/10.30880/ijie.2020.12.05.022

Received 16 April 2020; Accepted 15 June 2020; Available online 30 June 2020

\begin{abstract}
Epoxy composite has been widely used in various industrial applications due to its high strength. Nevertheless, its high strength causes it to fracture easily. Fillers are often added to improve its fracture toughness and other properties. In this work, epoxy composites containing carbon black (CB) and graphene have been synthesized to assess their mechanical properties. Series of analysis have been performed on composites containing single filler (CB or graphene) of various loadings to evaluate the values of Young's Modulus, yield strength, and KIC. The results demonstrate an improvement in Young's Modulus, yield strength, and KIC values by adding different carbon fillers, compared to neat epoxy. Based on these results, composites containing dual fillers are fabricated to understand the resultant synergistic effects. The hy-brid fillers show an increment in both fracture toughness and yield strength test for the epoxy composite with an optimum improvement at (3.0 wt.\% CB + 0.1 wt.\% Graphene) loading which is an increase of $256 \%$ in the values of Young's Modulus, compared to neat epoxy. The addition of carbon fillers enhances the mechanical properties of epoxy composites, with dual fillers demonstrate the highest improve-ment, which could be due to the improvement in the dispersion degree.
\end{abstract}

Keywords: Carbon Black, fracture toughness, graphene, yield strength, Young's Modulus

\section{Introduction}

Due to its exceptional mechanical properties, epoxy composite has been widely used in various areas including aerospace, structural, automotive or electrical components. Epoxy with a higher crosslink density contributes to low absolute strength and poor fracture toughness, limiting its applications in most industrial applications [1]. In an effort to improve various mechanical properties of the epoxy, different types of fillers were added. Types of fillers added include silica [2- 3], CNT [4-5], graphene [6-7], carbon black [8-9] and clay [10]; among others. These studies reported an enhancement in the mechanical properties of epoxy.

Graphene is, basically, a single atomic layer of graphite; an abundant mineral which is an allotrope of carbon that is made up of very tightly bonded carbon atoms organized into a hexagonal lattice. What makes graphene so special is its sp2 hybridization and very thin atomic thickness. These properties are what differentiate graphene to be highly distinguished than another compound [11]. In recent researches, an increased attention has been seen in graphene nanoplates and graphene nanoparticles as possible nanofillers for nanocomposite materials [12]. It is reported in literature that the fracture toughness of the nanocomposite starts to decrease beyond certain weight fraction [13]. As the filler content increases, KIC also increases for low filler content $(0-0.5 \mathrm{wt} \%)$ and for higher weight fraction of GNP (more than $1.0 \mathrm{wt} \%$ ), a decreasing trend is observed as reported in [13]. As the graphene content has a loading of $0.1 \mathrm{wt} \%$ into neat epoxy resin, the fracture toughness values in-creases up to $128 \%$ [14]. For higher weight fraction of graphene nanoplatelets, GNP which is a composition of stacked 2-D graphene sheets, (more than $1.0 \mathrm{wt} \%$ ), a decreasing trend is observed. It was discovered that the fracture toughness value starts decreasing for GNP/epoxy at $1.0 \mathrm{wt} \%$ [13]. The 
incorporated nanosized fillers are usually selected according to the desired properties of the final composite. A research done by [15] on the comparison of fracture performance of epoxy composites with $0.1 \%$ wt of pure graphene, single walled and multi walled carbon nanotubes finds that the composite which present with graphene has the highest fracture toughness among the composites. At low nanofiller content, graphene nanocomposite depicts significant improvement in mechanical properties when compared to CNT. A report shown on the fracture toughness of epoxy polymer reinforced with various weight percentage of graphene. Remarkably, only at loading $0.125 \%$ of graphene shows an increment of fracture toughness of pristine epoxy by $65 \%$. Fractography analysis indicated that impressive effectiveness of graphene ability to resist fracture is interrelated to the deflection process which associated with the planar (two dimensional) structure of one-dimensional CNTs or low-aspect-ratio nanoparticles. Drastic improvement can be noticed from the addition of $0.125 \mathrm{wt} \%$ of graphene into the epoxy matrix at $1.75 \mathrm{MPa} \cdot \mathrm{m}^{1 / 2}$ which corresponds to a $65 \%$ increase in fracture toughness. The higher loading weight percentage profound to be a diminishing factor for enhancement in fracture toughness which begins to approach the neat epoxy value at loading

$0.5 \mathrm{wt} \%$. This might be a result of degradation in the dispersion of the FGS additives above a weight fraction of $0.125 \%$. The Young's Modulus of the nanocomposite also project a 50\% increase from baseline epoxy with the UTS being increased by $45 \%$ both at loading of $0.125 \mathrm{wt} \%$ [16].

The results of the tensile tests showed that adding graphene oxide, GO increased the tensile strength, load, Young's modulus and elongation. The fracture strain was seen to decrease [17]. The loading of $0.1 \mathrm{wt} \%$ graphene shows a significant value for tensile strength (78 MPa) which is $40 \%$ larger than pristine epoxy (55 MPa) thus far outperform Single Walled Carbon Nanotube (SWNT) and Multi Walled Carbon Nanotube (MWNT) at $11 \%$ and $14 \%$ increase. Regarding to the fact that it was achieved at nanofiller weight fraction of $0.1 \mathrm{wt} . \%$ is remarkable. The incorporation of $0.1 \mathrm{wt} \%$ loading of graphene increases the Young's Modulus from neat epoxy at $31 \%$ increment from 2.85 GPa to 3.74 GPa while the increment of SWNT and MWNT both yield a lower increment at $(<3 \%)$. Fracture toughness also shows a significant increment for $0.1 \mathrm{wt} \%$ graphene filled epoxy at $53 \%$ improvement from neat epoxy. The fracture toughness of GPL is also impressive in relation to nanoparticle composites [12]. In summary, graphene is tremendously effective at reinforcing the fracture energy, stiffness, strength, fracture toughness, and fatigue resistance of epoxy polymers at significantly lower nanofiller loading fractions [16].

Previous studies have shown promising results in improvement of mechanical properties of epoxy composites as a result of incorporating $\mathrm{CB}$ and graphene. It is interesting to find out the synergistic effects demonstrated by incorporating these two fillers. There are also several studies that reported improvement of mechanical properties of composites from utilizing dual fillers. For example, it was reported that the incorporation of $\mathrm{CB}$ as a second filler accordingly enhanced the plasticity and fracture strength properties of the epoxy-CNT-CB hybrid nanocomposite while having the same high strength and flexural modulus [18]. The enhancement of strength properties of hybrid composite mainly regards to the fact that homogeneous distribution of the filler particles in the epoxy matrix and the formation of a bond between the matrix and the fiber surface at the interface [19]. The introduction of small amount of CNTs or GNPs to CB/polymer composites can significantly enhanced their properties. An excellent synergistic effect was also observed for flexural modulus measurements where the composites with CNT:GnP ratio of 9:1 showed the highest increase of 17\% com-pared to an increase of $9 \%$ and 5\% with pure CNT and GnP respectively. The flexural modulus for the CNT:GnP ratio of 5:1 was also observed to be higher than that of composites with pure CNT and GnP [20]. The carbon-graphene epoxy nanocomposite with 0.1 wt. $\%$ fracture toughness indicate $11.4 \%$ enhancement at (33.55 MPa.m ${ }^{1 / 2}$ ) in comparison with traditional carbon-epoxy compo-sites. Although, neat epoxy exceeds the improvement of fracture toughness from this finding, it is evident that the gains observed represent a partial enhancement over carbon-epoxy composites [14].

\section{Materials and Method}

The epoxy used in this experiment was CP812, supplied by Orential Option Sdn Bhd. Both graphene and CB were purchased from China. The carbon materials were added to the epoxy resin at the specified weight loading $(0.1$ wt. $\%, 0.5$ wt. $\%, 1.0 \mathrm{wt} . \%, 1.5 \mathrm{wt} . \%, 2.0 \mathrm{wt} . \%$, and $3.0 \mathrm{wt}$.) and stirred at $1000 \mathrm{rpm}$, for 15 minutes. Next the hardener was added to the mixture (at 1:2 weight ratio) and stirred at the same speed for 5 minutes. The mixture was then left to cure in a mould for a day at room temperature. The size of the mould depends on the type of mechanical test to be performed later. FESEM was used to characterize the structure of CB and graphene. Prior to that, the particles were slightly pressed onto adhesive car-bon (Agar Scientific), which was placed on a 0.5" pin stub (Agar Scientific). The FESEM used in this work is Gemini 500 from Carl Zeiss, operated at $5 \mathrm{kV}$. INSTRON 5569A Universal Testing Machine was used to conduct tensile test, in accordance to ASTM D638. The test was operated at a constant rate of $2 \mathrm{~mm} / \mathrm{min}$ with a $20 \mathrm{kN}$ load cell. Fracture toughness (KIC) was evaluated by using the three-point end notch bending (SENB) test mode, which has the load cell capacity of $10 \mathrm{kN}$ based on ASTM5045. The fracture test was also conducted by using INSTRON 5569A Universal Testing Machine 


\section{Results and Discussion}

Fig. 1 shows the Field Emission Scanning Electron (FESEM) images of the Carbon Black (a) and Graphene (b). CB appears to be in no defined shape while the structure of graphene is in flakes form. Both of them are in nanoparticle size and highly agglomerated, which are due to Van der Waals attraction.

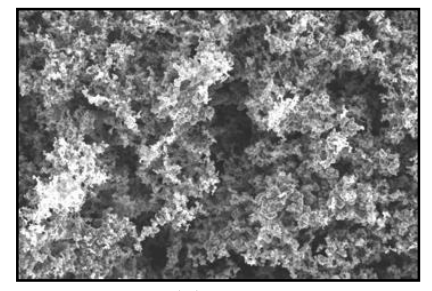

(a)

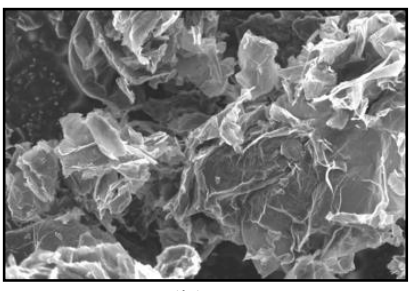

(b)

Fig. 1 - FESEM images of (a) Carbon Black and (b) Graphene, viewed at 10,000 magnifications

Fig. 2 shows the $\mathrm{K}_{\mathrm{IC}}$ values for epoxy composite containing individual CB and graphene fillers Fig. 1 (a). In the case of $\mathrm{CB}$ fillers, there is a decrease in fracture toughness for loading at $(0.1 \mathrm{wt} . \%-1.5 \mathrm{wt}$. \%). A significant rise in the fracture toughness is observed by incorporating the carbon black into the epoxy resin for loadings range between $2.0 \mathrm{wt}$. $\%$ till 4.0 wt. \% loading. For samples containing 0.1 wt. \% carbon black, the values of $\mathrm{K}_{\mathrm{IC}}$ is close to neat epoxy. These were caused by the low percentage of filler in the epoxy resin that resulted the composite to retain its original mechanical properties thus retaining its fracture toughness value. The value for $0.5 \mathrm{wt}$. $\%$ shows a drop in fracture toughness at $31 \%$ reduction. For loading at $1.0 \mathrm{wt}$. \% also shows a $34 \%$ decrease in fracture toughness from neat epoxy. It is also similar for loading $1.5 \mathrm{wt}$. \% having a $34 \%$ decrease in fracture toughness at $0.41913 \mathrm{MPa}$. A slight curving trend can be observed from the increment of loading at $3.0 \mathrm{wt} \%$ being the most significant increase in fracture toughness, $\left(\mathrm{MPa} \cdot \mathrm{m}^{1 / 2}\right)$. The result of $2.0 \mathrm{wt} \%$ shows a $12 \%$ reduction in fracture toughness at $0.55884 \mathrm{MPa}$ but it is being the starting point for the increasing trend for the loading of the composite. The highest improvement at $81 \%$ was observed for Epoxy-CB composite from loading $3.0 \mathrm{wt} \%$ which is followed by a drop in $4.0 \mathrm{wt} \%$ loading thus making it the optimum improvement for the composite.

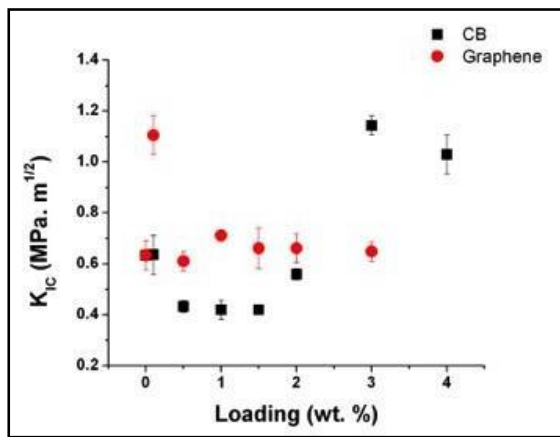

(a)

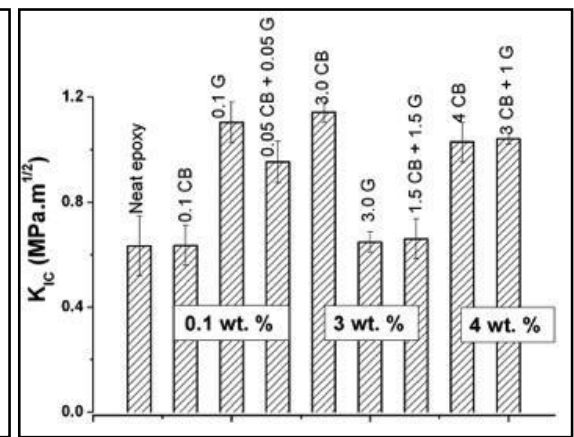

(b)

Fig. 2 - (a) $K_{I C}$ values for epoxy composite containing individual $C B$ and graphene fillers and (b) $K_{I C}$ values for epoxy composite containing dual fillers, with single filler also shown for comparison

In the case of graphene, an increase in $\mathrm{K}_{\mathrm{IC}}$ can be observed at loading (1.0 wt. \% till $\left.3.0 \mathrm{wt} . \%\right)$. The most significant improvement is determined at 0.1 wt. $\%$ loading. A significant increase in $75 \%$ in fracture toughness $(1.11$ MPa. $\mathrm{m}^{1 / 2}$ ) is observed at loading of $0.1 \mathrm{wt} \%$. As the weight percentages increases, the graph shows a minor fluctuation trend on the fracture toughness as shown in Fig. 2 (a). However, at loading 0.5 wt. \% loading, a reduction of $3.7 \%$ is observed compared to neat epoxy. For $1.0 \mathrm{wt} \%$ loading, the result shows a $12 \%$ increase in fracture toughness. A minor fluctuation increase can be seen for loadings of $1.5 \mathrm{wt} \%$ and $2.0 \mathrm{wt} \%$, which is an increment for $4.4 \%$ from neat epoxy. Loading at $3.0 \mathrm{wt} \%$ also shows a minor increase of $2.3 \%$ from neat epoxy. This is due to the high loading that converged the epoxy resin to become more ductile but low in fracture strength. Neat epoxy in general is a brittle but strong material but when mixed with other enhancing filler it can reform its structure either becoming more brittle or ductile. In this case, it is evident that the epoxy resin has become more ductile and less brittle due to the fact that the specimen tends to bend excessively and not break fully when tested.

As shown in Fig. 2 (right) the combined loading of $(0.05$ wt. $\% \mathrm{CB}+0.05$ wt. $\% \mathrm{G})$ shows a $51 \%$ increase of fracture toughness from neat epoxy. When compared to fracture toughness of single fillers $0.1 \mathrm{wt}$ \% Epoxy-CB, the hybrid composite $(0.05$ wt. $\% \mathrm{CB}+0.05 \mathrm{wt} \% \mathrm{G})$ shows an increase at $50 \%$ and a decrease at $0.1 \mathrm{wt} \%$ but depicts a drop compared to 0.1 wt. $\%$ Epoxy-G at $16 \%$. A minor drop can be seen on the hybrid loading of 0.1 wt. $\%$ of both 
CB-G when analyzed to lower hybrid loading of (0.05 wt. \% CB + 0.05 wt. \% G). However, a $42 \%$ improvement in fracture toughness can be seen from neat epoxy and also increases at $109 \%$ and $48 \%$ when compared to loading 0.5 wt. $\% \mathrm{CB}$ and 0.5 wt. \% G. Fig. 2 (right) shows at (3.0 wt. \% CB + 0.1 wt. \% G) it is evident that it yields the highest value for fracture toughness among all hybrid fillers composite at $1.0415 \mathrm{MPa} \cdot \mathrm{m}^{1 / 2}$ which is an increase of $64 \%$ from neat epoxy. It also indicated a $1.2 \%$ enhancement when compared to $4.0 \mathrm{wt}$. \% CB composite and $60 \%$ increment from $0.1 \mathrm{wt}$. \% G loading. The loading at (1.5 wt. \% CB + $1.5 \mathrm{wt}$. \% G) shows just a slight increase of 0.6605 $\mathrm{MPa}^{1 / 2}$ from neat epoxy at $4.3 \%$ and $2 \%$ from $3.0 \mathrm{wt} \%$ Epoxy-CB. Although, the result also shows a $42 \%$ drop compared to the single fillers of $3.0 \mathrm{wt} \%$ Epoxy-G.

Fig. 3 shows the Young's Modulus obtained from tensile test. A similar trend for the values of Young's Modulus (with fracture toughness test is observed for loading (0.5 wt. \% - $2.0 \mathrm{wt} \%$ ) and an increase for loading (3.0 wt. \% - 4.0 wt. \%), for composites containing CB. The result obtained for Young's Modulus of loading at 0.1 wt. \% shows a slight increase from neat epoxy at $0.4 \%$. For $0.5 \mathrm{wt}$. \% shows a $43 \%$ decrease from neat epoxy at $1405.46 \mathrm{MPa}$. Loading at $1.0 \mathrm{wt} . \%$ also shows a $37 \%$ reduction from neat epoxy. The lowest value can be seen on loading $1.5 \mathrm{wt}$. $\%$ which have a $49 \%$ decrease from neat epoxy. The result also shows a significant increase of $98 \%$ Young's Modulus at loading 3.0 wt. \% (Fig. 3 (a)). It can be concluded that 3.0 wt. \% is the ultimate improvement in enhancing the fracture toughness of the epoxy composite containing CB. Thus, a similar trend can be seen on research done by previous studies that recorded improvement in strength properties by increasing the loading.

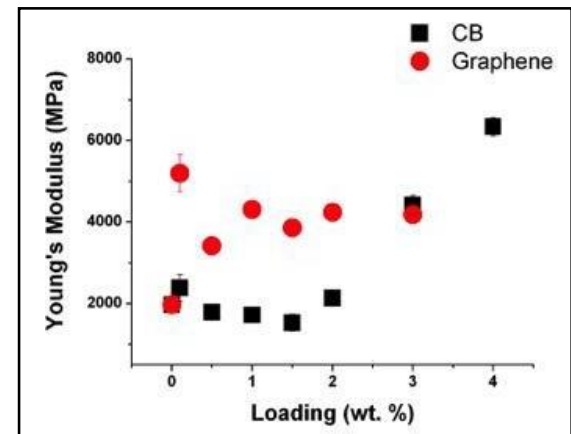

(a)

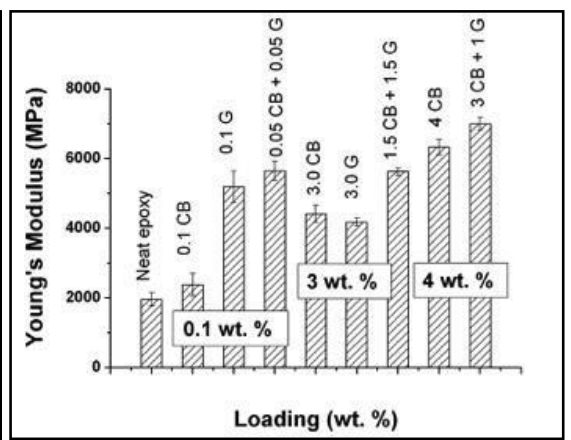

(b)

Fig. 3 - (a)Young's Modulus values for epoxy composite containing individual CB and graphene fillers; (b)Young's Modulus values for epoxy composite containing dual fillers, with single filler also shown for comparison

Young's Modulus value of epoxy-graphene composite as shown in Fig. 3 (a) depicts an improvement with the increase of loading in the range of $1.0 \mathrm{wt}$. \% till $3.0 \mathrm{wt}$. \%. A significant drop at $0.5 \mathrm{wt}$. \% graphene loading was observed that caused a decrease of $8.4 \%$ in the values of Young's Modulus, compared to neat epoxy. The loading at 0.1 wt. \% shows a high improvement for the Young's Modulus at 132\% increase from neat epoxy. An increase from neat epoxy at $161 \%$ is observed for $1.0 \mathrm{wt}$. \% loading of graphene. A high enhancement of graphene fillers for epoxy is obtained at 1.5 wt $\%$ and $2.0 \mathrm{wt} \%$ which has a percentage increase of $170 \%$ and $182 \%$ from neat epoxy. The highest improvement can be identifying at graphene loading of $3.0 \mathrm{wt} \%$ that has a value of $7458.09 \mathrm{MPa}$ in which is an increment from neat epoxy at about $205 \%$.

The result for Young's Modulus for dual fillers are as shown in Fig. 3 (b). The highest yield is shown for the combined loading of (3.0 wt. \% CB + $0.1 \mathrm{wt} . \% \mathrm{G})$ that has a Young's Modulus increase of $151 \%$ from neat epoxy and also an $88 \%$ compared to $4.0 \mathrm{wt}$ \% $\% \mathrm{CB}$, however a reduce of $17 \%$ from single fillers $3.0 \mathrm{wt}$. \% G. Hybrid composite at loading $(0.05$ wt. $\% \mathrm{CB}+0.05$ wt. $\% \mathrm{G})$ depicts a Young's Modulus increase of $64 \%$ and $66 \%$ from $0.1 \mathrm{wt}$. \% CB single filler and neat epoxy. However, a $29 \%$ drop can be determined from measuring with hybrid epoxy from $0.1 \mathrm{wt}$. \% G single filler. For loading at $(1.5 \mathrm{wt} \% \mathrm{CB}+1.5 \mathrm{wt} \% \mathrm{G})$, the Young's Modulus can be identifying as an increment from both neat epoxy at percentage increase of $68 \%$ but not more than 0.1 wt. \% Epoxy-G and 3.0 wt. \% Epoxy-CB single filler which is $44 \%$ and $15 \%$ much higher than the hybrid composite.

Fig. 4 shows the values of yield strength for both individual fillers (a) and dual fillers (b). An increase the values of yield strength for loading $\mathrm{CB}$ at $1.0 \mathrm{wt} \%$ can be seen before reduces at $1.5 \mathrm{wt}$. $\%$. The loading at $0.1 \mathrm{wt} \%$ shows a reduction of $22.4 \%$ from neat epoxy. A similar but slightly higher value of yield strength for neat epoxy is shown at loading of 0.5 wt. $\%$ which has an increase of $0.38 \%$. An increase can be identifying for $1.0 \mathrm{wt} \%$ loading which have a percentage gain of $27 \%$ from neat epoxy. However, the results value then drops at $1.5 \mathrm{wt} \%$ and $2.0 \mathrm{wt} \%$ with a percentage decrease of $35 \%$ and $14 \%$ of yield strength compared to neat epoxy. Next, $3.0 \mathrm{wt} \%$ loading when referred to neat epoxy, depicts a rise in yield strength at 35\% increase. The loading at $4.0 \mathrm{wt} \%$ have the most significant improvement in yield strength, which have a value of $39.20 \mathrm{MPa}$ that is about $78 \%$ increment from neat epoxy. It is evident that by increasing the loading for the carbon black filler, the enhancement of epoxy composite will increase with minor fluctuation throughout the result. 


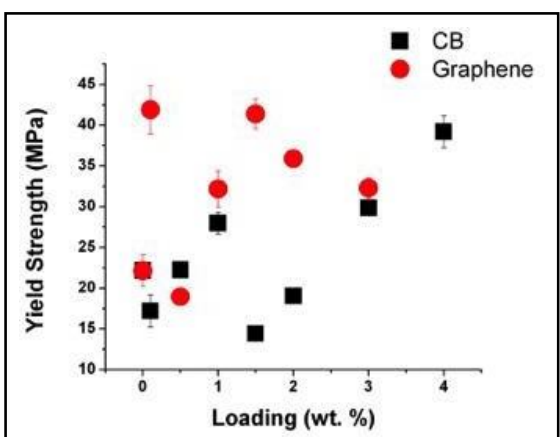

(a)

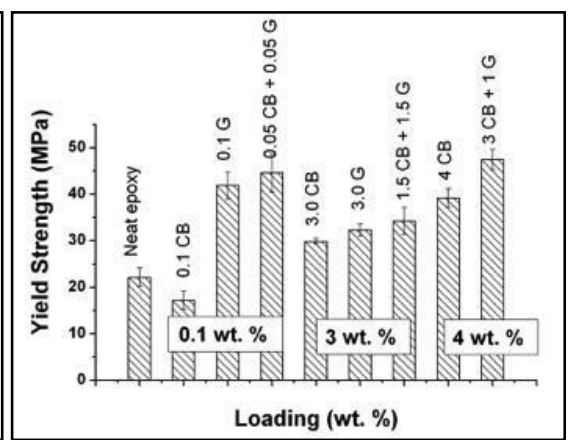

(b)

Fig. 4 - (a) Yield strength values for epoxy composite containing individual CB and graphene fillers; (b) yield strength values for epoxy composite containing dual fillers, with single filler also shown for comparison

Fig. 4 (a) also shows the result of yield strength obtained from Epoxy-Graphene composite. The highest improvement for the epoxy composite in yield strength is at $0.1 \mathrm{wt}$. \% loading which has an increment of $89 \%$ from neat epoxy at 5197.23 MPa. The yield strength result drop at $0.5 \mathrm{wt}$. \%, which is $14.5 \%$ decrease from neat epoxy. A rising trend then occurred at $1.0 \mathrm{wt} . \%$ and $1.5 \mathrm{wt} . \%$ which is an increase of $45 \%$ and $87 \%$ from neat epoxy, respectively. This is followed up by descend at $3.0 \mathrm{wt} \%$ and $4.0 \mathrm{wt} \%$ which is still an increase of $62 \%$ and $46 \%$ compared to neat epoxy.

The yield strength portrays maximum improvement of $47.67 \mathrm{MPa}$ at $(3.0 \mathrm{wt}$ \% $\mathrm{CB}+0.1 \mathrm{wt}$ \% G) loading for hybrid epoxy composite as shown in Fig. 4 (b) The loading of (0.05 wt. \% CB + 0.05 wt. \% G) depicts a significant improvement from neat epoxy, $0.1 \mathrm{wt}$ \% CB and $0.1 \mathrm{wt}$. \% G composite at percentage increase of $101 \%, 159 \%$ and $6.4 \%$, respectively. Thus, this already proved to have a synergistic effect from the integration of the single filler composites. The yield strength can be seen on loading $(0.1 \mathrm{wt} . \% \mathrm{CB}+0.1 \mathrm{wt} . \% \mathrm{G})$ showed a tremendous enhancement compared to the single fillers and epoxy at average percentage increase of $98 \%$. Furthermore, the loading at (1.5 wt. \% CB + 1.5 wt. \% G) were justified and it also to have a synergistic effect on the epoxy considering the increment of 54\% and $15 \%$ from neat epoxy and $3.0 \mathrm{wt}$ \% $\%$ CB. Loading at $(1.5 \mathrm{wt} \% \mathrm{CB}+1.5 \mathrm{wt} \% \mathrm{G})$ also shows a slight increment of $6.1 \%$ from $3.0 \mathrm{wt}$. \% G. The highest increment is on the loading of $(3.0 \mathrm{wt} . \% \mathrm{CB}+0.1 \mathrm{wt} . \% \mathrm{G})$ which have a $21 \%$ improvement compared to the $4.0 \mathrm{wt}$ \% CB that is among the highest in yield strength for single fillers composite.

\section{Conclusion}

Epoxy composite containing various fillers fracture toughness and tensile strength were tested in this research and shows rather satisfying results when compared to previous studies. At the same wt.\%, composites containing hybrid showed higher values of Young's Modulus and Yield Strength, compared to the composites containing only single filler. In the case of fracture toughness, the value of $\mathrm{K}_{\mathrm{IC}}$ of at $1.0415 \mathrm{MPa} \cdot \mathrm{m}^{1 / 2}$ was obtained at $(3.0 \mathrm{wt} . \% \mathrm{CB}+0.1 \mathrm{wt} . \% \mathrm{G})$, which is an increase of $64 \%$ from neat epoxy. It is evident that the hybrid composite does found as an improvement for most single fillers, only certain loading second towards the optimum result value of single filler epoxy composite. The enhancement of strength properties of hybrid composite mainly regards to the fact that homogeneous distribution of the filler particles in the epoxy matrix and the formation of a bond between the matrix and the fiber surface at the interface [19].

\section{Acknowledgement}

The research acknowledges the Universiti Pertahanan Nasional Malaysia and Ministry of Higher Education, Malaysia and RACE/F2/TK/UPNM/1 for financial support.

\section{References}

[1] Zaman, I., et al. (2011), Epoxy/graphene platelets nanocomposites with two levels of interface strength. Polymer, 52, 1603-1611.

[2] Blackman, B.R.K., et al., (2007). The fracture and fatigue behaviour of nano-modified epoxy polymers. Journal of Materials Science, 42, 7049-7051.

[3] Ma, J., et al (2008)., Effect of inorganic nanoparticles on mechanical property, fracture toughness and toughening mechanism of two epoxy systems. Polymer, 49, 3510-3523.

[4] Gojny, F.H., et al. (2005), Influence of different carbon nanotubes on the mechanical properties of epoxy matrix composites - A comparative study. Composites Science and Technology, 65, 2300-2313. 
[5] Zhang, X.-H., et al., (2008) Toughening of cycloaliphatic epoxy resin by multiwalled carbon nanotubes. Journal of Applied Polymer Science, 110, 1351-1357.

[6] Zainal, N., Arifin, H.H., Zardasti, L., Yahaya, N., Lim, K.S. and Md Noor, N. (2018) Mechanical Properties of Graphene-Modified Epoxy Grout for Pipeline Composite Repair. International Journal of Integrated Engineering, 10,4

[7] Potts, J.R., et al. (2011), Graphene-based polymer nanocomposites. Polymer, 52, 5-25.

[8] Kim, B.C., S.W. Park, and D.G. Lee (2008), Fracture toughness of the nano-particle reinforced epoxy composite. Composite Structures, 86, 69-77.

[9] Gojny, F.H., et al. (2004), Carbon nanotube-reinforced epoxy-composites: enhanced stiffness and fracture toughness at low nanotube content, Composites Science and Technology, 64, 2363-2371.

[10] Koh, K.L., et al. (2017), Fracture Toughness and Elastic Modulus of Epoxy-Based Nanocomposites with Dopamine-Modified Nano-Fillers. Materials, 10, 776-780.

[11] Zhang, G., J. Karger-Kocsis, and J. Zou, (2010) Synergetic effect of carbon nanofibers and short carbon fibers on the mechanical and fracture properties of epoxy resin. Carbon, 48, 4289-4300.

[12] Rafiee, M.A., et al. (2009), Enhanced Mechanical Properties of Nanocomposites at Low Graphene Content. ACS Nano, 3, 3884-3890.

[13] Chandrasekaran, S., et al. (2014). Fracture toughness and failure mechanism of graphene based epoxy composites. Composites Science and Technology, 97, 90-99.

[14] Tkachev, S.V., E.Y. Buslaeva, and S.P. Gubin (2011), Graphene: A novel carbon nanomaterial, Inorganic Materials, 47, 1-10.

[15] Papageorgiou, D.G., I.A. Kinloch, and R.J. Young (2015), Graphene/elastomer nanocomposites. Carbon, 95, 460484.

[16] Rafiee, M.A., et al. (2010). Fracture and Fatigue in Graphene Nanocomposites. Small, 6, 179-183.

[17] Cha, J., et al. (2017), Improvement of modulus, strength and fracture toughness of CNT/Epoxy nanocomposites through the functionalization of carbon nanotubes. Composites Part B: Engineering, 129, 169-179.

[18] Szeluga, U., B. Kumanek, and B. Trzebicka (2015), Synergy in hybrid polymer/nanocarbon composites. A review. Composites Part A: Applied Science and Manufacturing, 73, 204-231.

[19] Kumari, N. and K. Kumar, (2018) Mechanical behaviour of graphene and carbon fibre reinforced epoxy based hybrid nanocomposites for orthotic callipers. Journal of Experimental Nanoscience, 13, S14-S23.

[20] Chatterjee, S., et al. (2012), Size and synergy effects of nanofiller hybrids including graphene nanoplatelets and carbon nanotubes in mechanical properties of epoxy composites. Carbon, 50, 5380-5386.

[21] Schaeffer, W.D., W.R. Smith, and M.H. Polley (1953), Structure and Properties of Carbon Black - Changes Induced by Heat Treatment. Industrial \& Engineering Chemistry. 45, 1721-1725. 